OPEN ACCESS

Edited by:

Rick D'Eath,

Scotland's Rural College,

United Kingdom

Reviewed by:

Jen-Yun Chou,

University of Pennsylvania,

United States

Tamara Alejandra Tadich,

University of Chile, Chile

*Correspondence: Perle E. Zhitnitskiy pboyer@umn.edu

Specialty section:

This article was submitted to Animal Welfare and Policy,

a section of the journal

Frontiers in Animal Science

Received: 03 January 2021 Accepted: 22 February 2021

Published: 16 March 2021

Citation:

Zhitnitskiy PE, Terreaux CMHA Phillips HN and Ventura BA (2021) Short-Term Impact of Point-Source

Enrichment on the Behavior of Gestating Sows Housed in Groups.

Front. Anim. Sci. 2:649114.

doi: 10.3389/fanim.2021.649114

\section{Short-Term Impact of Point-Source Enrichment on the Behavior of Gestating Sows Housed in Groups}

\author{
Perle E. Zhitnitskiy ${ }^{1 *}$, Claire M. H. A. Terreaux ${ }^{2}$, Hannah N. Phillips ${ }^{3}$ and Beth A. Ventura ${ }^{3}$ \\ ${ }^{1}$ Department of Veterinary Population Medicine, College of Veterinary Medicine, University of Minnesota, Minneapolis, MN, \\ United States, ${ }^{2}$ Zoopole Développement, Centre Technique des Productions Animales, Ploufragan, France, ${ }^{3}$ Department of \\ Animal Science, College of Food, Agricultural, and Natural Resources, University of Minnesota, Minneapolis, MN, \\ United States
}

Environmental enrichment is an important strategy to improve the welfare of farm animals. However, relatively little is known about enrichment for gestating sows, especially those raised on farms with slatted floors and for which provision of straw may be difficult. The objective of this study was therefore to investigate the short-term (4 d) impact of a point-source enrichment object on the behavior of gestating sows housed in group pens. Four pens of gestating sows on a university research farm were randomly allocated to receive either enrichment or no enrichment (control) in a 2 by 2 crossover design. Time budgets were established by video recording focal sows' behaviors ( $n=10$ focals per pen) every 15 min between 0800 and 1500 every day. Enrichment use was further characterized by continuous behavior sampling for a $1 \mathrm{~h}$ interval between 0830 and 0930 each day. The impact of parity, lameness and presence of stereotypical behavior such as sham chewing on enrichment use was evaluated. Over the course of the study, focal sows spent approximately $73 \%$ of observations inactive [either lying down (70\%), standing $(2 \%)$, or sitting (1\%)]. Within the remaining observations, sows were most commonly observed sham-chewing (16\%), followed by $3 \%$ exploring, $2 \%$ feeding, $2 \%$ walking and $1 \%$ interacting with the enrichment when it was available. Low-parity sows, moderately-lame sows, and sows observed sham chewing at baseline displayed more consistent enrichment use over the course of the study $(p=0.02, p<0.01, p=0.04$, respectively). While no adverse behavioral effects (increased agonism or sham chewing) due to provision or removal of the enrichment object were observed and while $85 \%$ of sows were observed to interact with enrichment at least once, interest declined sharply after the first day. We conclude that further research is needed to identify effective and sustainable enrichment strategies for gestating sows.

Keywords: sow, behavior, enrichment object, stereotypies, group-housing 


\section{INTRODUCTION}

The housing of pregnant sows is one of many areas of livestock welfare that has elicited societal concern in recent decades. Until recently, most sows in the United States were housed in individual crates during their entire gestation period, without the possibility to turn around. Individual sow housing systems were designed to enable individualized attention to nutrition, health, reproduction, and management (McGlone et al., 2004). However, systems that prohibit postural movements also induce many welfare challenges (McGlone et al., 2004), including but not limited to the restriction of motivated behaviors to socialize, forage, and explore the environment. Such systems have been the focus of increased concern amongst members of the public (Ryan et al., 2015; Yunes et al., 2018). In response, numerous US states have passed voter-driven referenda to prohibit or phase-out the use of systems that prevent basic postural movement, while in the European Union, the Council Directive 2001/88/EC prohibits the individual housing of sows and gilts after the fourth week of gestation until the week preceding farrowing (Pedersen, 2018). To address increasing concerns about animal welfare, swine production companies and retailers have started to ask suppliers to transition toward group systems, which allow greater freedom of movement and social interaction with conspecifics (e.g., in early 2018, the largest pork producer in the world, Smithfield Foods, announced that they had transitioned all company-owned farms to group housing of pregnant sows apart from breeding and farrowing, Smithfield Foods Company).

Transitioning to group systems can represent a significant challenge for farmers, partially because housing sows in groups can introduce new challenges to swine welfare. For example, sows must establish social hierarchies within the group to function well (Hunter et al., 1988; Greenwood et al., 2014) and aggressive behaviors are common within the first $48 \mathrm{~h}$ after sows are mixed together. Additionally, such aggression can persist if sow social dynamics are managed inappropriately (e.g., frequent mixing of groups), compromising sow welfare and production (Verdon and Rault, 2018).

However, strategic changes in the existing environment through environmental enrichment have the potential to improve sow well-being. Enrichment has been defined by Newberry (1995) as an improvement in an animal's biological functioning through modifications to the environment. Successful enrichment should result in some or all of the following outcomes: (1) increased expression (i.e., frequency and range) of normal, motivated behaviors, (2) reduction or prevention of abnormal behaviors, (3) improved use of available space, and (4) improved abilities to cope with challenges associated with the captive environment (Mkwanazi et al., 2019). While the science of enrichment for growing pigs is extensive, research relative to gestating sows is limited (Van De Weerd and Ison, 2019). Of the enrichment approaches explored for gestating sows, most have sought to stimulate the expression of normal foraging and oral behaviors or to improve the structural complexity and division of the available pen space (Stewart et al., 2008; Van de Weerd and Day, 2009; Horback et al., 2016).
Provision of straw is considered an important component of gold-standard enrichment for pigs, as it is destructible, manipulatable, and edible (Van De Weerd and Ison, 2019), but straw can pose management challenges for many commercial swine facilities. In such cases, various forms of point-source enrichment objects may be useful in providing an outlet for some of the most important motivated behaviors in pigs, such as foraging and other oral behaviors. EasyFix Lunas are made of natural rubber and are designed to encourage play and oral behaviors in pigs (EasyFix, EasyFix). While Lunas may have a mild positive effect in growing pigs (generating more interaction overall and reducing harmful behaviors during the weaner stage compared to spruce wood posts (Chou et al., 2020a), there is little published research on their effects in gestating sows. While there does not appear to be consensus on what constitutes "short-term" when investigating impacts of enrichment, an evaluation of 74 different enrichment objects given to growing pigs showed that a duration of five days was sufficient to identify which enrichments failed to sustain interest in the animals (Van De Weerd et al., 2003). Additionally, Elmore et al. (2011) observed agonistic behaviors in sows within the hour following introduction of enrichment.

Therefore, the objectives of this study were to: (1) assess the short-term impact of providing point-source object enrichment on the behavior of gestating sows housed in groups (with attention to stereotypic and agonistic behaviors), (2) describe sows' interactions with this enrichment and, (3) evaluate the association between sow parity, sham chewing, and lameness status with enrichment interaction.

\section{MATERIALS AND METHODS}

The animal protocol was reviewed and approved by the University of Minnesota Institutional Animal Care and Use Committee under the reference 1811-36558A.

\section{Animal Care and Provision of the Enrichment \\ Facility and Animal Management}

This study was conducted at the University of Minnesota Southern Research and Outreach Center 750-head swine unit in Waseca, Minnesota in April 2019. This farrow-to-wean farm was managed in a batch farrowing schedule, such that the herd was divided into 10 groups of sows, one of them farrowing every other week. Sows were comprised of Landrace and Yorkshire breeds and were gestating their first to tenth litter. The facility housed sows in gestation stalls from weaning until they were confirmed pregnant for approximately 35 days. At that time, sows were grouped into pens. Sows were fed via a single electronic sow feeder per pen and water was available ad libitum via four nipple drinkers per pen. Feed rations were individualized based on body condition and gestation stage. Sows were relocated to individual farrowing stalls in a farrowing room during the week prior to their expected farrowing date. The flooring of the gestation room consisted of concrete slats above deep manure pits. Manure pit exhausts and wall fans ensured active ventilation 


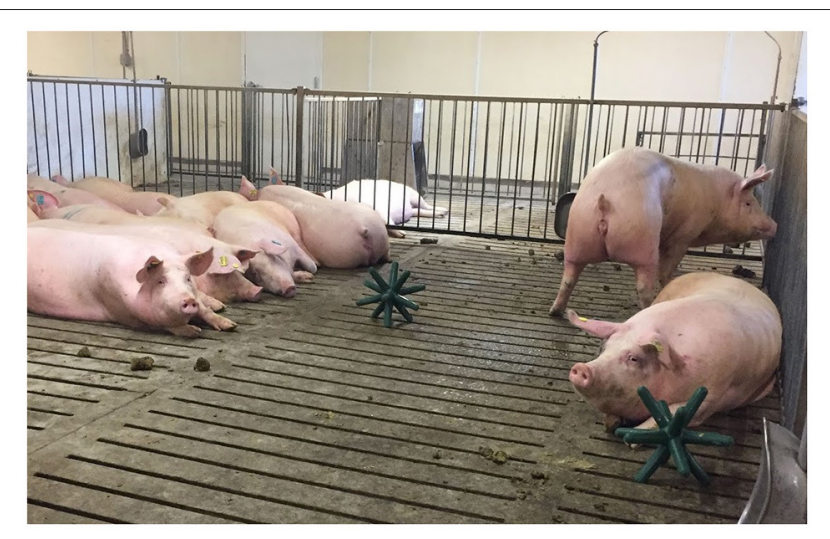

FIGURE 1 | Picture of the enrichment objects (Luna) in the pen.

of the room by creating negative air pressure. The barn was temperature-controlled (between 21 and $23^{\circ} \mathrm{C}$ ) and lighting schedule was $9 \mathrm{~h}$ light: $15 \mathrm{~h}$ dark. At the time of the study, each pen (dimensions $6.88 \mathrm{~m}$ by $13.11 \mathrm{~m}$ ) housed between 44 and 53 sows, with stocking densities ranging from 1.70 to $2.05 \mathrm{~m}^{2}$ per sow.

\section{Experimental Design}

The enrichment evaluated in this study was a point-source object called Luna 142 (Figure 1), designed as a food-grade durable rubber star-shaped toy to stimulate oral and investigatory behaviors in adult pigs (EasyFix, Galway, Ireland). Four pens out of the six available in the gestation room were enrolled in this study. The two remaining pens were not included because they either housed non-pregnant gilts or sows due to be moved into farrowing rooms during the experiment. In a cross-over design, the pens were randomly allocated to receive the enrichment object at a ratio of at least one per nine sows during either the first period ( $\mathrm{d} 1-4$, group 1 ) or the second period (d5-8, group 2 ) of the study (Table 1). Once placed, enrichments remained in the pen until the end of the period to allow sows continuous access. Enrichment objects were cleaned and disinfected between treatment periods.

\section{Focal Sows}

Behaviors were recorded for 10 randomly selected focal sows per pen (40 sows in total). The list of sows' identification numbers was randomized in the 2016 Excel software (Microsoft Corporation, Redmond, WA) and the first 10 sows in each pen were selected for behavioral observations. Each of the selected focal sows were scored for lameness based on a previously described protocol (Performance Zinpro Swine Locomotion Scoring, Feet First Scoring System, Zinpro Performance Minerals) where sows were scored between 0 and 3. Any sow scoring above 2.5 (defined as: "Lameness is involved in one or more limbs. The sow exhibits compensatory behaviors such as dipping her head or arching her back") was not considered for behavioral observations and the next sow on the randomly generated list was evaluated. Focal sows were designated with numbers between 1 and 10, written on their backs and flanks with a livestock marker to allow for visual identification during video observations.

\section{Data Collection}

Eight cameras (two per pen) were installed on the ceiling or walls surrounding pens. Cameras captured video footage for a total of nine days including day 0 (baseline) before enrichment was added. Video footage of the pens was recorded daily from 0800 until 1500, when the lights were on in the gestation room and when farm staff were less likely to be in the room for morning and late afternoon checks.

To obtain estimates of time budgets (Whalin et al., 2016), four observers recorded behaviors for each of the focal sows every 15 min between day 0 and day 8 , yielding 29 observations per sow per day (261 total behavior scans per sow over the study period). Training of observers was considered sufficient after at least $90 \%$ agreement between observers for 120 scans. Table 2 presents the ethogram of behaviors recorded for this study.

Continuous behavior sampling for enrichment use was also performed by two observers each day from 0830 to 0930 . This time period, directly after the daily reset of the electronic sow feeders and the sows' first feeding, was chosen based on expected greater activity levels of sows during the morning, which was hoped to optimize the observation of enrichment object use. The intra-class correlation coefficient was computed to assess agreement between the observers in recording total daily duration and daily total bouts of enrichment use in all focal sows for five of the nine experimental days (100 observations). There was high absolute agreement between observers, using the twoway random effect models and single rater unit, kappa $\geq 0.94$, $p<0.01$. Statistical analysis showed no significant difference in behaviors recorded by observers.

\section{Statistical Analyses Time Budget}

Data from the behavioral scans were used to construct a time budget for the following behaviors: standing inactive, lying inactive, walking, exploring, enrichment use, sham chewing, sitting, agonistic behaviors, and other. Agonistic behaviors, including vulva biting, knocking over, levering, biting, mounting, chasing, disturbing, and displacing, were aggregated into a single behavior for analysis. All other behaviors, including scratching, elimination, human interaction, positive or neutral social interactions, were aggregated due to low frequencies of observation. For each behavior outcome, daily pen means were calculated by summing the number of observations that sows were recorded performing that behavior outcome and dividing by the number of complete observations, resulting in a single daily non-Bernoulli binomial value per pen. The total possible number of daily observations per pen was 290 (29 observations for 10 focal sows). However, some observations were missing because of either camera recording issues or poor visibility of focal sows, which resulted in a different number of complete observations for each pen. Seventeen percent of daily pen observations were missing on average (range 3$34 \%)$. 
TABLE 1 | Crossover design of the study.

\begin{tabular}{|c|c|c|c|c|}
\hline & & Baseline (day 0) & Period 1 (day 1 to 4 ) & Period 2 (day 5 to 8 ) \\
\hline \multirow[t]{2}{*}{ Group 1} & Pen A, 48 sows & No enrichment & 6 enrichment objects & No enrichment \\
\hline & Pen $B, 53$ sows & No enrichment & 6 enrichment objects & No enrichment \\
\hline \multirow[t]{2}{*}{ Group 2} & Pen C, 44 sows & No enrichment & No enrichment & 5 enrichment objects \\
\hline & Pen D, 53 sows & No enrichment & No enrichment & 6 enrichment objects \\
\hline
\end{tabular}

TABLE 2 | Experimental ethogram for gestating sows.

\begin{tabular}{|c|c|c|}
\hline Behavior & Analysis label & Description \\
\hline Standing inactive & "Standing inactive" & Assuming or maintaining an upright position on four extended legs. \\
\hline Lying inactive & "Lying inactive" & $\begin{array}{l}\text { Lying with the majority ( }>50 \% \text { ) of the sternum contacting the ground or lying with either the left or right side of the } \\
\text { body in contact with the ground. }\end{array}$ \\
\hline Walking & "Walking" & 4-beat locomotion in which legs propel sow's movement across the pen. \\
\hline Running & N/A & 4-beat locomotion which is faster than a walk, for more than $1 \mathrm{~s}$. \\
\hline Exploration & "Exploring" & Rooting, sniffing, touching the pen floor or pen elements with snout or mouth. \\
\hline Enrichment use & "Enrichment" & $\begin{array}{l}\text { Snout is directed toward Luna object and sow is sniffing or contacting the toy (e.g., pushing, biting, or picking up } \\
\text { and tossing). }\end{array}$ \\
\hline Sham chewing & "Sham chewing" & $\begin{array}{l}\text { Oral stereotypic behavior in which sow repeatedly chews without having anything in her mouth. Sow may be in } \\
\text { any position. }\end{array}$ \\
\hline Dog-sitting & "Sitting" & Sitting on the hind end with forelegs stretched out to support the body. \\
\hline Downward dog & "Sitting" & $\begin{array}{l}\text { Forelegs are in contact with the floor with the hind legs in a standing position, lifting the rump. Sow may be } \\
\text { inactive or in transition from stand to lay. }\end{array}$ \\
\hline Vulva biting & "Agonistic" & Biting another pig's perineal region once or repeatedly, or receiving the biting. \\
\hline Knock & "Agonistic" & Knocks another sow using her head and neck, contacting any part of the receiving sow, or receiving the knock. \\
\hline Lever & "Agonistic" & Head is used to lift or attempt to lift any other part of the body of another sow, or is being levered. \\
\hline Bite & "Agonistic" & One single bite delivered from one sow to any part of another (except the perineal region), or receiving the bite. \\
\hline Mount & "Agonistic" & One sow mounts another, with her front legs both over the back of another animal, or is mounted. \\
\hline Chase & "Agonistic" & $\begin{array}{l}\text { Sow pursues another sow who is attempting to move quickly away from her, or sow who moves quickly away to } \\
\text { escape another sow chasing her. }\end{array}$ \\
\hline Disturb & "Agonistic" & $\begin{array}{l}\text { Sow walks over other sows in search of place to rest or in transition to another area of the pen; or sow is lying } \\
\text { down and is walked over, causing her to upset herself. }\end{array}$ \\
\hline Displace & "Agonistic" & $\begin{array}{l}\text { Movement of one sow by another such that the sow gives way to the other without her having to use physical } \\
\text { contact, or movement away in response to another sow's approach or presence. }\end{array}$ \\
\hline Other social & "Other" & $\begin{array}{l}\text { All other neutral or positive social behaviors in which sows actively direct snouts toward each other, especially } \\
\text { toward the face, but actions are not aggressive or submissive. }\end{array}$ \\
\hline Scratching & "Other" & Sow vigorously rubs her body against pen object \\
\hline Elimination & "Other" & Defecation or urination \\
\hline Human interaction & "Other" & $\begin{array}{l}\text { Workers walk the pens once/day for animal health checks/heat detection/breeding. Human interaction recorded } \\
\text { when focal sow is observed }<1 \text { meter from human. }\end{array}$ \\
\hline Other behaviors & "Other" & All other behaviors, or behavior cannot be clearly defined \\
\hline Unknown ${ }^{1}$ & "Other" & Sow is not visible on camera/occluded and behavior cannot be determined or the camera has malfunctioned \\
\hline
\end{tabular}

${ }^{1}$ For observations in which a sow was occluded or not visible on camera, or camera malfunctioned, observations were removed and excluded from analysis.

Data were analyzed using logistic regression models with binomial error distributions and logit link functions using the glmmTMB function of the glmmTMB package (Brooks et al., 2017) in RStudio (version 1.3.1073, R Core Team). Models were built for each behavior outcome with a covariate for baseline measures, fixed factors of treatment (2 levels: control and enrichment), day (4 levels: day 1-day 4), the treatment and day interaction, period (2 levels; period 1: day 1-4 and period 2: day 5-8), and order in which pens received the enrichment
(2 levels: first or second). Pen within treatment (8 levels) was a random subject intercept and the weight statement included the total number of complete observations. The baseline covariate was removed if the corresponding Wald Chi-square test had $p>0.05$. Baseline covariates were included to analyze behaviors of exploring, sham chewing, standing inactive, and walking. The model for the analysis of enrichment interaction included only the fixed factors of order and day, and the random subject intercept was pen (4 levels). Furthermore, a subset of the full 
TABLE 3 | Characteristics of focal sows (number out of $n=10$ focal sows).

\begin{tabular}{ccccccccc}
\hline & \multicolumn{2}{c}{ Lameness group } & & \multicolumn{2}{c}{ Parity group } & & \multicolumn{2}{c}{ Sham chewing prevalence } \\
Pen & Low & Moderate & & Low & High & & Absent & Present \\
\hline A & 4 & 5 & & 5 & 5 & & 4 & 6 \\
B & 5 & 5 & & 6 & 4 & & 2 & 8 \\
C & 7 & 3 & & 5 & 5 & & 5 & 5 \\
D & 3 & 7 & 5 & 5 & & 5 & 5 \\
\hline
\end{tabular}

dataset was used that only included data for when the enrichment was present in the pen. The weighted arithmetic mean was used to determine means and $90 \%$ CIs for baseline behaviors.

\section{Continuous Enrichment Use}

Data from 1-h observations of sows were aggregated for each focal sow and day. Sows were divided into low $(0-2 ; n=21)$ or high $(3-9 ; n=19)$ parity groups, low $(0-1 ; n=19)$ or moderate $(1.5-2.5 ; n=20)$ lameness groups, and absence $(n=$ $16)$ or presence $(n=24)$ of baseline sham chewing (i.e., whether sows had been observed to sham chew on the baseline day) groups to investigate the effects of parity, lameness, and baseline sham chewing behaviors on enrichment use outcomes (Table 3). One sow was removed from this analysis because of a missing lameness score.

Zero-inflated negative binomial linear mixed models analyzed duration of enrichment interaction per hour and the number of enrichment interaction bouts per hour using the $\operatorname{glmm} T M B$ function. A similar model that excluded the zero-inflation factor was used to analyze durations of bouts. Fixed effects included day (4 levels), period (2 levels), parity (2 levels), lameness (2 levels), and sham chewing (2 levels), the parity and day interaction, the lameness and day interaction, and the sham chewing and day interaction. Random effects were included for sow (39 levels) and pen (4 levels).

For all analyses, the significance of main effects was tested by obtaining the $p$-value for the Wald Chi-square test and declared when $p \leq 0.05$. Pairwise comparisons were carried out only when effects had $p \leq 0.05$. Pairwise comparisons were made using odds ratios (OR) for outcomes analyzed on the logit-link scale (binomial) and rate ratios (RR) for outcomes analyzed on the loglink scale (negative binomial). If the Wald Chi-square test for a two-way interaction with day had $p \leq 0.05$, then daily predicted means for each treatment are reported, else the overall treatment means are reported. The results of the analyses are reported as back-transformed predicted means and $90 \%$ CIs. The Tukey $p$ value adjustment was applied for multiple pairwise comparisons of days.

\section{RESULTS}

\section{Time Budget}

Overall, focal sows spent $\sim 73 \%$ of observations inactive [either lying down (70\%), standing (2\%), or sitting (1\%)]. Within the remaining observations, sows were most commonly

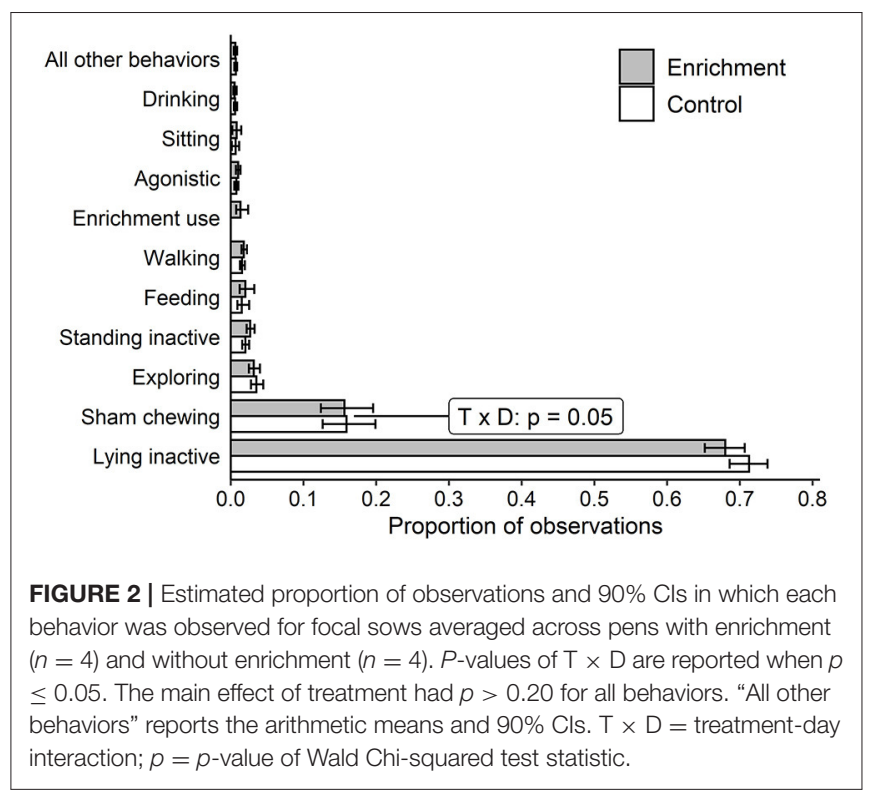

observed sham-chewing (16\%), followed by $3 \%$ exploring, $2 \%$ feeding, $2 \%$ walking, and $1 \%$ interacting with the enrichment when it was available (Figure 2). Sows were rarely observed performing agonistic behaviors, drinking or other behaviors $(<3 \%$ collectively).

No behavior was affected by treatment alone. Period and order effects were not detected for any analyses of behaviors, except for exploring such that the odds of exploring in period 2 was $1.6(90 \%$ CI $1.2-2.3, p=0.02$ ) times the odds of exploring in period 1.

Additionally, day of study had an effect on the lying inactive $\left[\chi^{2}{ }_{(3)}=18.5, p<0.01\right]$, standing inactive $\left[\chi^{2}{ }_{(3)}=17.6, p<0.01\right]$, and enrichment use behaviors $\left[\chi^{2}{ }_{(3)}=40.9, p<0.01\right]$, while a treatment and day interaction was detected for sham chewing $\left[\chi^{2}{ }_{(3)}=7.9, p=0.05\right]$. Daily predicted means for these behaviors are illustrated in Figure 3. No effect of day nor treatment and day interaction was found for the remaining behaviors: exploring, feeding, walking, agonistic, sitting, and drinking.

\section{Inactive Postural Behaviors of Lying and Standing}

The main effect of day was the only source of variation in the model to predict both lying inactive and standing inactive behaviors. With respect to lying inactive, sows on day 2 had 1.5 (90\% CI 1.2-1.7, $p<0.01$ ) and 1.3 (90\% CI 1.1-1.5, $p<0.01$ ) times greater odds of lying inactive compared to sows on days 1 and 4, respectively. Sows on day 3 had 1.4 (90\% CI 1.2-1.6, $p<0.01)$ times greater odds of lying inactive compared to sows on day 1 (Figure $3 \mathbf{A})$.

In terms of standing inactive, sows on day 1 had $2.3(90 \%$ CI 1.3-4.0, $p<0.01)$ and $1.8(90 \%$ CI 1.1-3.0, $p=0.05)$ times greater odds of performing this behavior compared to sows on day 2 and 3, respectively. Sows on day 4 had 2.2 (90\% CI 1.3$3.9, p<0.01)$ times greater odds of standing inactive compared to sows on day 2 (Figure 3B). Ultimately, both standing inactive and lying inactive behaviors were expressed similarly across days in the control and enrichment treatment groups. 

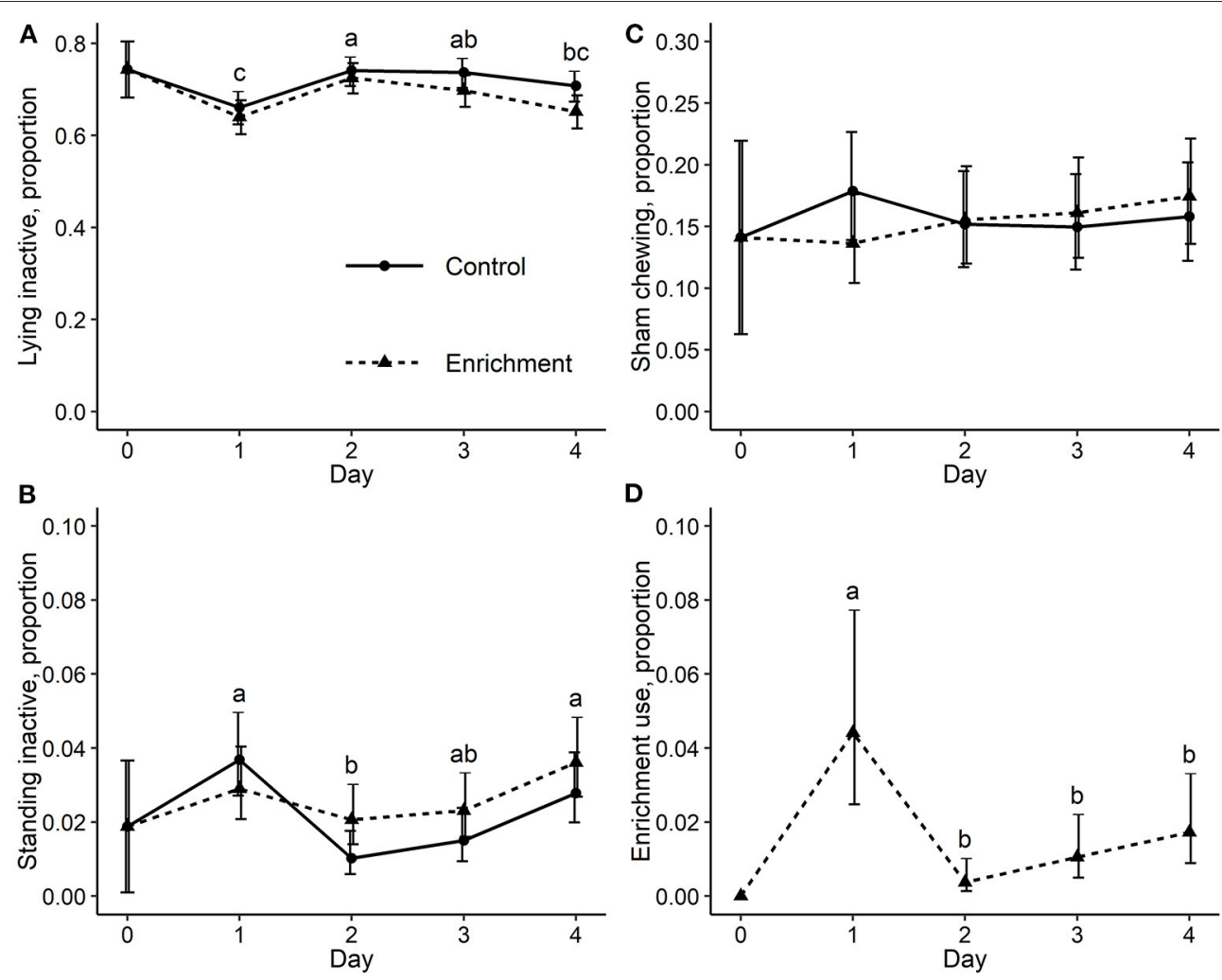

FIGURE 3 | Proportions and 90\% Cls of observations recording (A) lying inactive, (B) standing inactive, (C) sham chewing, and (D) enrichment use behaviors. a-C, days averaged between treatment groups (plots $\mathbf{A}-\mathbf{C}$ ) and days (plot $\mathbf{D}$ ) without a common letter have different means at $p \leq 0.05$.

\section{Sham Chewing}

Observations of sham chewing were affected by the interaction between treatment and day $(p=0.05)$, such that a numerically greater proportion of control sows were observed sham chewing on day 1 compared to sows receiving enrichment (Figure 3C).

\section{Enrichment Use}

\section{Contribution to Time Budget}

Enrichment use by sows varied over the days of the study $(p<0.01)$. The odds of enrichment use on day 1 was $12.4(90 \%$ CI 3.3-46.0, $p<0.01$ ), 4.3 (90\% CI 1.9-10.1, $p<0.01$ ), and 2.6 (90\% CI 1.3-5.3, $p=0.02$ ) times the odds of enrichment use on days 2, 3, and 4, respectively (Figure 3D).

\section{Sow Use of Enrichment}

Because of the sporadic nature of the enrichment use, sow behavior was further analyzed through daily 1-h continuous observations. Fifteen percent of the focal sows were never observed interacting with the enrichment during these observation intervals. Over half (55\%) of the focal sows were observed using the enrichment at least two of the four observation days, whereas $8 \%$ of focal sows interacted with enrichment every day of the study. On average, sows spent less than a minute interacting with enrichment within the 1-h observation interval, although the rate of interaction varied greatly among individuals (ranging from 0 to $18.7 \mathrm{~min}$ per $\mathrm{h}$ ).
Enrichment use (i.e., the number and duration of enrichment bouts, and total duration of use) was not affected by study period but was affected by study day and sow characteristics (e.g., parity, sham chewing, and lameness status). Daily least squares means for bouts of enrichment use for groups with different sham chewing prevalence are presented in Figure 4, and the daily least squares means for duration and number of bouts of enrichment use for groups with different lameness scores are presented in Figure 5. For all other variables, the two-way interactions with day were not significant.

\section{Parity}

Over the course of an hour of continuous observation, low-parity sows spent significantly more time interacting with enrichment $(60 \mathrm{~s} / \mathrm{h})$ compared to high-parity $(21 \mathrm{~s} / \mathrm{h})$ sows $(p=0.02)$. Furthermore, low-parity sows interacted with the enrichment at a rate of three bouts/h on average, whereas high parity sows interacted with enrichment at a rate of 1 bout/h $(p=0.01)$. There was no difference in the amount of time spent interacting with the enrichment for each of the bouts (Table 4).

\section{Sham Chewing}

The interaction between sham chewing and day impacted the rate of enrichment use bouts $\left[\chi^{2}{ }_{(3)}=8.1, p=0.04\right]$, such that sows observed sham chewing at baseline had a more consistent 


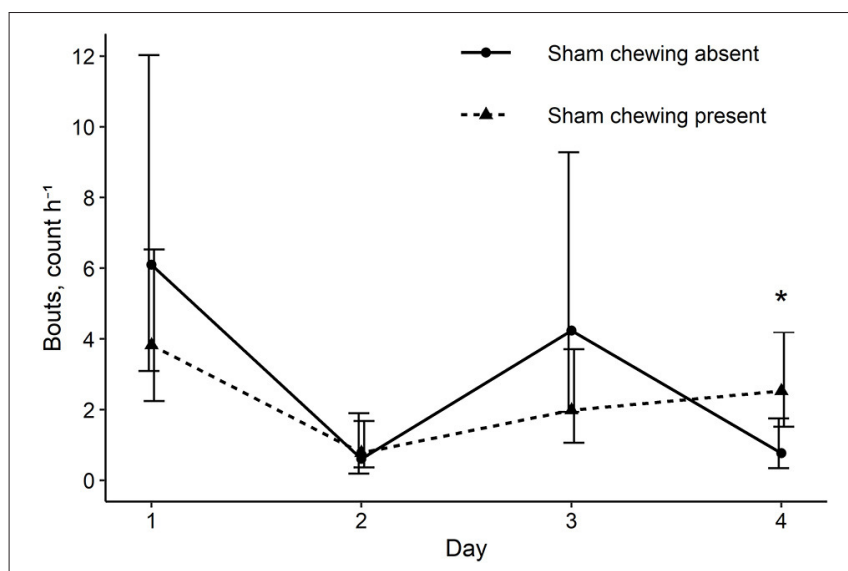

FIGURE 4 | Back transformed means and 90\% Cls for the frequency of enrichment bouts observed during the 1-h observation of sows with and without sham chewing. ${ }^{*}$ Means within a day are different at $p \leq 0.05$.

number of enrichment use bouts over the study, compared to sows who were not observed to sham chew at baseline (Figure 4).

\section{Lameness}

There was an interaction between lameness and day for the analysis of total duration of enrichment use $\left[\chi_{(3)}^{2}=19.5\right.$, $p<0.01]$ and number of bouts $\left[\chi^{2}{ }_{(3)}=9.2, p=0.03\right]$. On the first day of enrichment provision, total duration of enrichment use and number of bouts were similar across sows regardless of their lameness status. After day 1, moderately lame sows spent more time with enrichment compared to sows with low lameness (Figure 5A). Moderately lame sows also had more frequent bouts on day 2 of the study compared to sows with lower lameness scores (Figure 5B). Effectively, moderately lame sows appeared to more consistently retain enrichment interaction behaviors across the course of the study compared to relatively sound sows, whose enrichment use declined after day 1.

\section{DISCUSSION}

Finding environmental enrichment that engages gestating sows, avoids increased competition pressure, and is workable for farmers remains a challenge for the swine industry. For many farmers (e.g., those raising sows on fully slatted floors, or those facing tight economic margins), complex and varied enrichment schedules may be beyond reach. Thus, our objective was to discern whether provision of point-source enrichment designed to stimulate oral manipulation would sustain sow interest beyond the initial day of provision, as pigs may lose interest toward enrichment after the novelty wears off (Ernst et al., 2018). Previous work showed that nursery and finishing pigs were highly and sustainably interested in interacting with the same enrichment objects used in the current study (Chou et al., 2019, 2020a,b). Additionally, provision of these enrichment objects decreased the frequency of harmful behaviors including tail and ear biting in nursery pigs compared to spruce posts (Chou et al., 2020a), though that protective effect was not seen when the
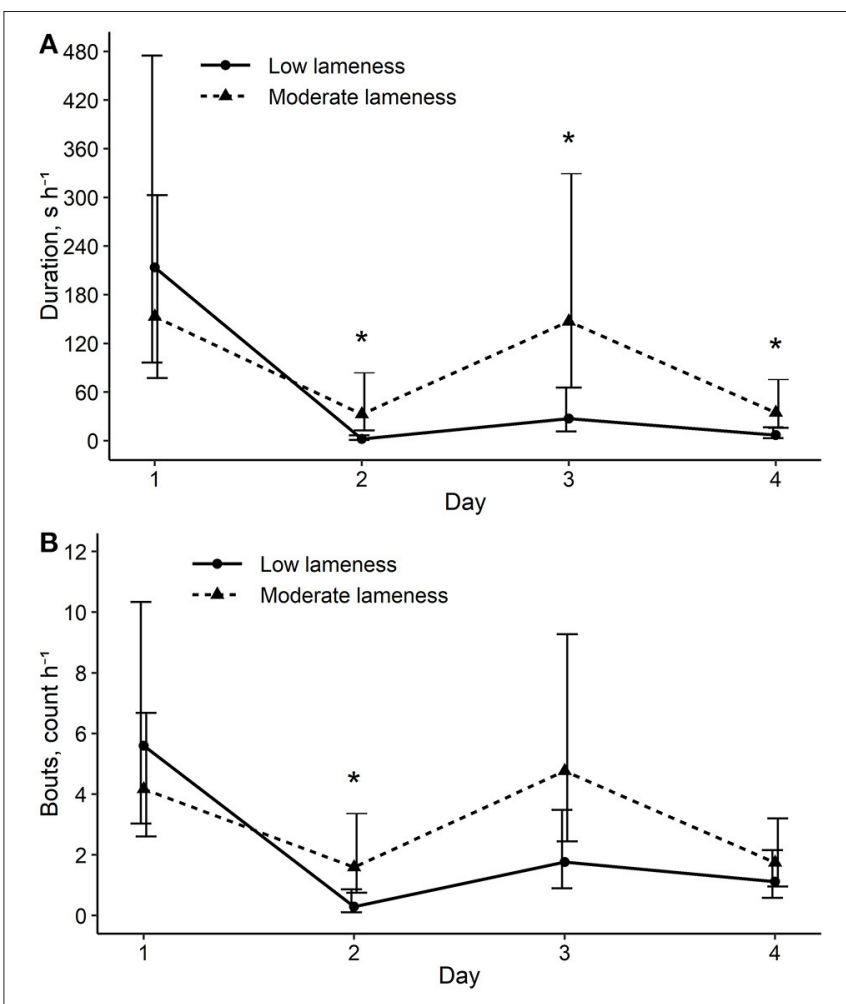

FIGURE 5 | Back transformed means and 90\% Cls comparing sows in low and moderate lameness score groups for outcomes of (A) total duration of enrichment interaction during the 1-h observation interval and (B) frequency of enrichment interaction bouts during the 1-h observation interval. * Means within a day are different at $p \leq 0.05$.

enrichment objects were provided to older pigs (Chou et al., 2020b). In contrast, while our results suggest that the Lunas did not introduce new behavioral problems in our population of gestating sows (i.e., no differences in agonistic or sham chewing behaviors between treatments), interest seemed to decline among most of the sows after the first day of provision.

\section{Time Budget}

\section{Sham Chewing}

The time budget analysis showed that lying inactive and sham chewing were the two most prevalent behaviors observed amongst focal sows in the present study. Sham chewing is a stereotypic behavior commonly observed in swine raised in intensive rearing systems (Rushen, 1985). Stereotypies are defined as invariant, repetitive, and apparently functionless behaviors and are generally considered to signal impaired animal welfare (Mason, 1991; Mason and Latham, 2004). Low feeding levels and behaviorally restrictive or barren environments have been shown to increase the prevalence of sham chewing behaviors in sows (Terlouw et al., 1991; Vieuille-Thomas et al., 1995). This study population was reared in a system characterized by these limitations; as part of the management of this particular facility, sows were feed-restricted to maintain body condition according to industry standards and spent several weeks in individual 
TABLE 4 | Least squares means and 90\% Cls for sows according to parity group and baseline sham chewing prevalence.

\begin{tabular}{|c|c|c|c|c|c|c|}
\hline \multirow[b]{2}{*}{ Behavior } & \multicolumn{2}{|c|}{ Parity } & \multicolumn{2}{|c|}{ Sham chewing } & \multicolumn{2}{|c|}{ Lameness } \\
\hline & Low & High & Absent & Present & Low & Moderate \\
\hline Total duration, s/h & $60^{a}(33,110)$ & $21 \mathrm{~b}(11,40)$ & $36(18,70)$ & $35(19,63)$ & - & - \\
\hline Number of bouts, count/h & $3^{a}(2,5)$ & $1^{b}(1,2)$ & - & - & - & - \\
\hline Duration of bouts, s/bout & $15(12,19)$ & $12(9,16)$ & $12(9,17)$ & $14(11,18)$ & $9(7,13)$ & $18(14,23)$ \\
\hline
\end{tabular}

-, $\chi^{2}$ had $p \leq 0.05$ for the interaction with day.

$a-b$, sows within a group and behavior with different letters have different means at $p \leq 0.05$.

gestation stalls before being moved into group pens for the remainder of their gestation periods. The proportion of time that sows were observed sham chewing was not affected by provision of the enrichment objects, an effect that may be explained by the short amount of time that most sows spent engaging with them. While some enrichment studies have failed to reduce sham chewing in sows [e.g., sows provided with access to a straw rack showed no difference in sham chewing compared to those without access (Stewart et al., 2008)], other enrichment strategies have successfully reduced observations of this behavior [e.g., farrowing and lactating sows provided with alfalfa hay showed reductions in sham chewing (Edwards et al., 2019)].

\section{Lying Inactive}

Proportions of time spent lying inactive differed by day, regardless of whether sows were provided with enrichment or not. Sows were most often observed lying inactive on days 2 and 3 , compared to days 1 and 4 . This pattern might be explained by the initial interest in the enrichment objects and the subsequent rebound sows displayed, respectively, on the first and fourth days of enrichment provision. These results may also indicate that activity levels were influenced by sow behavior and/or presence of enrichment in adjacent pens. This is not surprising, as pens were separated by bars and sows could see, hear, and engage in nose contact with neighbors. Finishing pigs have been shown to synchronize their behavioral expression, including enrichment use (Zwicker et al., 2015). Alternatively, this effect could be explained by emotional contagion, which has been demonstrated in pre-weaned (Goumon and Špinka, 2016), nursery (Reimert et al., 2017), and finishing pigs (Reimert et al., 2013). Those studies showed that pigs' behaviors were affected by positive or negative events administered directly to them and that control pigs-not subjected to those same events-nonetheless displayed similar behavioral and emotional states.

\section{Agonistic Behavior}

Aggression is a major welfare concern in group-housed sows (Maes et al., 2016), though it tends to be most problematic in the first few hours or days after social groups have been adjusted (Verdon and Rault, 2018). Incidence of aggression is influenced by many factors, including restriction of foraging and rooting, behavioral motivations which animals then redirect to conspecifics (Godyń et al., 2019). Environmental enrichment, as a (theoretically) desirable resource, can increase competition pressure (Van De Weerd and Ison, 2019), especially if individuals must compete for access to enrichment, for example if the enrichment is provided in insufficient quantities or is otherwise not accessible to all members of the group (Van de Weerd et al., 2006). Indeed, higher levels of aggression between conspecifics have been observed upon enrichment provision (Stewart et al., 2008; Roy et al., 2019). In the present study and similarly to Greenwood et al. (2018), neither provision nor removal of the point-source enrichment had any impact on expression of agonistic behavior among focal sows. However, this observation might be the result of the low prevalence of agonistic behaviors observed in the population studied.

\section{Enrichment Use}

Though the majority of focal sows (85\%) interacted with the enrichment on day 1 , their interest in enrichment had decreased sharply by the second day. Sows are more likely to remain interested in point-source enrichment if a rotation between objects of different natures is provided (Roy et al., 2019). However, sows' interest in the enrichment rebounded slightly after the second day of enrichment provision, a pattern consistent with another study in which sow play behaviors were highest 4 , 7 , and 20 days after the day of enrichment provision (Greenwood et al., 2018).

Lower parity sows spent more time interacting with the enrichment object as indicated by an increase in the number of enrichment interaction bouts. One hypothesis that could explain this phenomenon is that younger sows' behavior is closer to juvenile play than adult drive to interact with the enrichment, increasing their interest in the enrichment object (Greenwood et al., 2014). Alternatively, the hierarchy status of the sows may have played a role in the varying levels of enrichment use between younger and older sows. Although hierarchy status of the sows was not recorded in this study, there is evidence that lower parity sows tend to be subordinate until they reach their fourth parity, after which point body weight seems to be a stronger factor in determining status (Hoy et al., 2009). While both dominant and subordinate sows show the same motivation to access enrichment, a previous study suggests that dominant sows interact more with the enrichment when it is first presented to the group whereas subordinate sows interact thereafter (Elmore et al., 2011). The provision of point-source enrichment did not increase competition between sows in our study as demonstrated by the consistency in recorded agonistic behaviors across treatments. Here at least, the motivation to interact with the enrichment object was not strong enough to 
upset the social hierarchy in place in the study groups. This is in alignment with a previous study evaluating the impact of three different enrichment objects in a commercial sow herd (Horback et al., 2016). Therefore, the effect of parity on the level of enrichment interaction may be influenced by both age and hierarchy status.

Based on our results, lameness status may be associated with day-to-day fluctuations in the intensity of enrichment use, such that lamer sows seemed to have a prolonged interest in enrichment after the first day of provision. It is possible that less mobile sows are more limited within their behavior repertoire, as they might be reluctant to stand up, walk or explore unless motivated by food. Therefore, if an enrichment object is available and in close proximity (i.e., it does not require movement on their part to reach it) these sows may spend more time dedicated to enrichment interaction. Alternatively, engaging with enrichment may help sows attenuate their experience of pain brought on by lameness. Applications of environmental enrichment to alleviate pain have been explored in other animal models, perhaps most commonly rodents (Gabriel et al., 2010; Wang et al., 2019). For example, one study demonstrated that an enriched environment (social housing, shelter, running wheel, and marbles) reduced sensitivity to cold and mechanical stimuli in mice subjected to a spared nerve injury compared to mice housed singly in barren housing (Vachon et al., 2013). Piglets provided with object and substrate enrichment in the farrowing environment (rope, ball, newspaper, and soil) demonstrated improved growth $24 \mathrm{~h}$ after processing (castration, tail docking), and increased activity; however, enrichment did not reduce pain behaviors nor cortisol response after processing, leading the authors to conclude that enrichment improved piglet welfare but not necessarily pain experience per se (Backus and McGlone, 2018). Whether these differences are attributable to the nature of the pain (acute vs. chronic inflammation), age, species, nature, and timing of enrichment or previous experience is unclear, but this area warrants further investigation.

\section{Limitations}

This study examined the behaviors of a population of sows on a single farm for a limited duration of time and as such the results described here should be taken in context. There would be value in repeating this experiment to follow a different population of sows in other slatted floor facilities to strengthen understandings of how and if sows retain interest in these enrichment objects over a longer period of time. Other populations of sows characterized by lower incidences of sham chewing or lameness, for example, may interact differently with the point-source enrichment examined here. Lastly, there were a few times during the study that the enrichment objects became trapped in the electronic sow feeders and required workers to remove them. This is cause for concern as sows could become trapped within or blocked from accessing the feeders, so caretakers are cautioned that this type of point-source enrichment may require additional oversight to implement safely and effectively.

\section{CONCLUSION}

The objective of this study was to identify if point-source enrichment objects, seen as practical and easy from a management standpoint, would be biologically relevant to gestating sows housed in group pens. We first sought to confirm that provision of enrichment objects did not induce additional competition pressure or other unforeseen negative effects (e.g., increased sham chewing or aggression). From that standpoint, the enrichment objects were a success, as no additional adverse behavioral effects were observed, though we note that the enrichment objects may become trapped within electronic sow feeders. However, the majority of focal sows failed to sustain interest in the enrichment past the first day of provision. Van De Weerd et al., 2003; Van De Weerd and Ison, 2019 emphasized that effective enrichment for pigs should be investigable, manipulatable, destructible, and edible. Our results thus contribute to the growing consensus that effective enrichment strategies for pigs, including gestating sows, should focus on a combination of materials, including those which may be destroyed. The challenge remains to identify a compromise between what is best for pigs and what remains achievable for farm managers facing facility or management constraints.

\section{DATA AVAILABILITY STATEMENT}

The datasets presented in this study can be found in the Data Repository for the University of Minnesota (DRUM) https://doi. org/10.13020/prrx-k004.

\section{ETHICS STATEMENT}

The animal study was reviewed and approved by University of Minnesota Institutional Animal Care and Use Committee (reference 1811-36558A).

\section{AUTHOR CONTRIBUTIONS}

$\mathrm{PZ}, \mathrm{CT}$, and $\mathrm{BV}$ contributed to conception and design of the study and collected the data. HP organized the database and performed the statistical analysis. PZ wrote the first draft of the manuscript. HP and BV wrote sections of the manuscript. All authors contributed to manuscript revision, read, and approved the submitted version.

\section{FUNDING}

This study was funded through the University of Minnesota College of Veterinary Medicine Educational Leadership Grant.

\section{ACKNOWLEDGMENTS}

The authors gratefully acknowledge the staff at the UMN Southern Research Outreach Facility for their help with this study. We also thank Jennifer Bracken (University College Dublin, Ireland) for her assistance with data collection. 


\section{REFERENCES}

Backus, B. L., and McGlone, J. J. (2018). Evaluating environmental enrichment as a method to alleviate pain after castration and tail docking in pigs. Appl. Anim. Behav. Sci. 204, 37-42. doi: 10.1016/j.applanim.2018.04.009

Brooks, M. E., Kristensen, K., van Benthem, K. J., Magnusson, A., Berg, C. W., Nielsen, A., et al. (2017). GlmmTMB balances speed and flexibility among packages for zero-inflated generalized linear mixed modeling. $R$ J. 9, 378-400. doi: 10.32614/RJ-2017-066

Chou, J. Y., D’Eath, R. B., Sandercock, D. A., and O’Driscoll, K. (2020a). Enrichment use in finishing pigs and its relationship with damaging behaviours: comparing three wood species and a rubber floor toy. Appl. Anim. Behav. Sci. 224:104944. doi: 10.1016/j.applanim.2020.104944

Chou, J. Y., Drique, C., Sandercock, D., D'Eath, R., and O’Driscoll, K. (2019). Rearing undocked pigs on fully slatted floors using multiple types and variations of enrichment. Animals 9:139. doi: 10.3390/ani9040139

Chou, J. Y., O'Driscoll, K., Sandercock, D. A., and D'Eath, R. B. (2020b). Can increased dietary fibre level and a single enrichment device reduce the risk of tail biting in undocked growing-finishing pigs in fully slatted systems? PLoS ONE 15:e0241619. doi: 10.1371/journal.pone.0241619

EasyFix Pig Toys | Luna 142 | Environmental Enrichment. Available online at: https://easyfix.com/product/pig-toys-luna-142/ (accessed November 20, 2020).

Edwards, L. E., Plush, K. J., Ralph, C. R., Morrison, R. S., R., Acharya, R. Y., et al. (2019). Enrichment with Lucerne Hay improves sow maternal behaviour and improves piglet survival. Animals 9:558. doi: 10.3390/ani9080558

Elmore, M. R., Pittman Garner, J. P., Johnson, A. K., Kirkden, R. D., Richert, B. T., and Pajor, E. A. (2011). Getting around social status: motivation and enrichment use of dominant and subordinate sows in a group setting. Appl. Anim. Behav. Sci. 133, 154-163. doi: 10.1016/j.applanim.2011.05.017

Ernst, K., Ekkelboom, M., Kerssen, N., Smeets, S., Sun, Y., and Yin, X. (2018). Play Behavior and Environmental Enrichment in Pigs. Available online at: https://www.wur.nl/upload_mm/e/f/b/6af2e2db-430e-4771-8f7d6f5b974eab5e_finalreportACT2060juli2018opwebsite.pdf (accessed November $25,2020)$

Gabriel, A. F., Paoletti, G., Della Seta, D., Panelli, R., Marcus, M. A. E., Farabollini, F., et al. (2010). Enriched environment and the recovery from inflammatory pain: social versus physical aspects and their interaction. Behav. Brain Res. 208, 90-95. doi: 10.1016/j.bbr.2009.11.015

Godyń, D., Nowicki, J., and Herbut, P. (2019). Effects of environmental enrichment on pig welfare-a review. Animals 9:383. doi: 10.3390/ani9060383

Goumon, S., and Špinka, M. (2016). Emotional contagion of distress in young pigs is potentiated by previous exposure to the same stressor. Anim. Cogn. 19, 501-511. doi: 10.1007/s10071-015-0950-5

Greenwood, E., van Wettere, W., Rayner, J., Hughes, P., and Plush, K. (2018). Provision point-source materials stimulates play in sows but does not affect aggression at regrouping. Animals 9:8. doi: 10.3390/ani9010008

Greenwood, E. C., Plush, K. J., van Wettere, W. H. E. J., and Hughes, P. E. (2014). Hierarchy formation in newly mixed, group housed sows and management strategies aimed at reducing its impact. Appl. Anim. Behav. Sci. 160, 1-11. doi: 10.1016/j.applanim.2014.09.011

Horback, K. M., Pierdon, M. K., and Parsons, T. D. (2016). Behavioral preference for different enrichment objects in a commercial sow herd. Appl. Anim. Behav. Sci. 184, 7-15. doi: 10.1016/j.applanim.2016.09.002

Hoy, S., Bauer, J., Borberg, C., Chonsch, L., and Weirich, C. (2009). Investigations on dynamics of social rank of sows during several parities. Appl. Anim. Behav. Sci. 121, 103-107. doi: 10.1016/j.applanim.2009.09.003

Hunter, E. J., Broom, D. M., Edwards, S. A., and Sibly, R. M. (1988). Social hierarchy and feeder access in a group of 20 sows using a computer-controlled feeder. Anim. Produc. 47, 139-148. doi: 10.1017/S0003356100037144

Maes, D., Pluym, L., and Peltoniemi, O. (2016). Impact of group housing of pregnant sows on health. Porcine Health Manag. 2:17. doi: $10.1186 / \mathrm{s} 40813-016-0032-3$

Mason, G. J. (1991). Stereotypies: a critical review. Anim. Behav. 41, 1015-1037. doi: 10.1016/S0003-3472(05)80640-2

Mason, G. J., and Latham, N. R. (2004). Can't stop, won't stop: is stereotypy a reliable animal welfare indicator? Anim. Welfare 13, S57-69.
McGlone, J. J., von Borell, E. H., Deen, J., Johnson, A. K., Levis, D. G., Meunier-Salaon, M., et al. (2004). Compilation of the scientific literature comparing housing systems for gestating sows and gilts using measures of physiology, behavior, performance, and health. Professional Anim. Sci. 20, 105-117. doi: 10.15232/S1080-7446(15)31285-7

Mkwanazi, M. V., Ndumiso Ncobela, C., Tapera Kanengoni, A., and Chimonyo, M. (2019). Effects of environmental enrichment on behaviour, physiology and performance of pigs - a review. Asian Aust. J. Anim. Sci. 32, 1-13. doi: $10.5713 /$ ajas.17.0138

Newberry, R. C. (1995). Environmental enrichment: increasing the biological relevance of captive environments. Appl. Anim. Behav. Sci. 44, 229-243. doi: 10.1016/0168-1591(95)00616-Z

Pedersen, L. J. (2018). "Overview of commercial pig production systems and their main welfare challenges," in Advances in Pig Welfare, ed M. Špinka (Cambridge, MA: Woodhead Publishing), 3-25. doi: 10.1016/B978-0-08-101012-9.00001-0

Performance Zinpro Swine Locomotion Scoring, Feet First Scoring System, Zinpro Performance Minerals. Available online at: https://www.zinpro.com/lameness/ swine/locomotion-scoring (accessed April 15, 2019).

Reimert, I., Bolhuis, J. E., Kemp, B., and Rodenburg, T. B. (2013). Indicators of positive and negative emotions and emotional contagion in pigs. Physiol. Behav. 109, 42-50. doi: 10.1016/j.physbeh.2012.11.002

Reimert, I., Stephanie Fong, S., Rodenburg, T. B., and Bolhuis, J. E. (2017). Emotional states and emotional contagion in pigs after exposure to a positive and negative treatment. Appl. Anim. Behav. Sci. 193, 37-42. doi: 10.1016/j.applanim.2017.03.009

Roy, C., Lippens, L., Kyeiwaa, V., Seddon, Y. M., Connor, L. M., and Brown, J. A. (2019). Effects of enrichment type, presentation and social status on enrichment use and behaviour of sows with electronic sow feeding. Animals 9:369. doi: 10.3390/ani9060369

Rushen, J. P. (1985). Stereotypies, aggression and the feeding schedules of tethered sows. Appl. Anim. Behav. Sci. 14, 137-147. doi: 10.1016/0168-1591(85) 90025-5

Ryan, E. B., Fraser, D., and Weary, D. M. (2015). Public attitudes to housing systems for pregnant pigs. PLoS ONE 10:e0141878. doi: 10.1371/journal.pone.0141878

Smithfield Foods Company. Housing of Pregnant Sows | 2016 Sustainability Report. Available online at: https://www.smithfieldfoods.com/integratedreport/2016/animal-care/housing-of-pregnant-sows (accessed December $23,2020)$.

Stewart, C. L., O'Connell, N. E., and Boyle, L. (2008). Influence of access to straw provided in racks on the welfare of sows in large dynamic groups. Appl. Anim. Behav. Sci. 112, 235-247. doi: 10.1016/j.applanim.2007.09.006

Terlouw, E. M. C., Lawrence, A. B., and Illius, A. W. (1991). Influences of feeding level and physical restriction on development of stereotypies in sows. Anim. Behav. 42, 981-991. doi: 10.1016/S0003-3472(05)80151-4

Vachon, P., Millecamps, M., Low, L., Thompsosn, S. J., Pailleux, F., Beaudry, F., et al. (2013). Alleviation of chronic neuropathic pain by environmental enrichment in mice well after the establishment of chronic pain. Behav. Brain Funct. 9:22. doi: 10.1186/1744-9081-9-22

Van De Weerd, H., and Ison, S. (2019). Providing effective environmental enrichment to pigs: how far have we come? Animals 9:254. doi: 10.3390/ani9050254

Van de Weerd, H. A., and Day, J. E. L. (2009). A review of environmental enrichment for pigs housed in intensive housing systems. Appl. Anim. Behav. Sci. 116, 1-20. doi: 10.1016/j.applanim.2008.08.001

Van De Weerd, H. A., Docking, C. M., Day, J. E. L., Avery, P. J., and Edwards, S. A. (2003). A systematic approach towards developing environmental enrichment for pigs. Appl. Anim. Behav. Sci. 84, 101-118. doi: 10.1016/S0168-1591(03)00150-3

Van de Weerd, H. A., Docking, C. M., Day, J. E. L., Breuer, K., and Edwards, S. A. (2006). Effects of species-relevant environmental enrichment on the behaviour and productivity of finishing pigs. Appl. Anim. Behav. Sci. 99, 230-247. doi: 10.1016/j.applanim.2005.10.014

Verdon, M., and Rault, J. R. (2018). "Aggression in group housed sows and fattening pigs," in Advances in Pig Welfare, ed M. Špinka (Cambridge, MA: Woodhead Publishing), 235-60. doi: 10.1016/B978-0-08-101012-9.ol0 0006-X 
Vieuille-Thomas, C., Le Pape, G., and Signoret, J. P. (1995). Stereotypies in pregnant sows: indications of influence of the housing system on the patterns expressed by the animals. Appl. Anim. Behav. Sci. 44, 19-27. doi: 10.1016/0168-1591(95)00574-C

Wang, X. M., Zhang, G. F., Jia, M., Xie, Z. M., Yang, J. J., Shen, J. C., et al. (2019). Environmental enrichment improves pain sensitivity, depression-like phenotype, and memory deficit in mice with neuropathic pain: role of NPAS4. Psychopharmacology 236, 1999-2014. doi: 10.1007/s00213-019-5187-6

Whalin, L., Pairis-Garcia, M., Proudfoot, K., Stalder, K., and Johnson, A. (2016). Validating behavioral sampling techniques for lame sows administered flunixin meglumine and meloxicam. Livestock Sci. 191, 103-107. doi: 10.1016/j.livsci.2016. 07.017

Yunes, M. C., Von Keyserlingk, M. A. G., and Hötzel, M. J. (2018). Restricting the ability of sows to move: a source of concern for some Brazilians. Anim. Welfare 27, 379-392. doi: 10.7120/09627286.27.4.379
Zwicker, B., Weber, R., Wechsler, B., and Gygax, L. (2015). Degree of synchrony based on individual observations underlines the importance of concurrent access to enrichment materials in finishing pigs. Appl. Anim. Behav. Sci. 172, 26-32. doi: 10.1016/j.applanim.2015.08.037

Conflict of Interest: The authors declare that the research was conducted in the absence of any commercial or financial relationships that could be construed as a potential conflict of interest.

Copyright (C) 2021 Zhitnitskiy, Terreaux, Phillips and Ventura. This is an open-access article distributed under the terms of the Creative Commons Attribution License (CC $B Y)$. The use, distribution or reproduction in other forums is permitted, provided the original author(s) and the copyright owner(s) are credited and that the original publication in this journal is cited, in accordance with accepted academic practice. No use, distribution or reproduction is permitted which does not comply with these terms. 